\title{
Interventions under Video-Augmented X-Ray Guidance: Application to Needle Placement
}

\author{
M. Mitschke ${ }^{1}$, A. Bani-Hashemi ${ }^{2}$, and N. Navab ${ }^{2}$ \\ 1 Siemens AG Medical Engineering, Henkestr. 127, 91052 Erlangen, Germany \\ 2 Imaging \& Visualization, Siemens Corporate Research, Princeton, NJ 08540-6632 \\ Matthias.Mitschke@med.siemens.de, $\{$ ali,navab\}@scr.siemens.com
}

\begin{abstract}
The camera augmented mobile C-arm (CAMC) has been introduced in 12] for the purpose of online geometrical calibration. Here, we propose its use for an augmented reality visualization. Introducing a double mirror system [1] the optical axes of both imaging systems (Xray and optical) can be aligned. With this property both images can be merged or co-registered by only a planar transformation. This allows a real-time augmentation of X-ray and CCD camera images. We show that the needle placement procedure can be performed under this augmented reality visualization instead of fluoroscopy. Only two single X-ray images from different unknown $\mathrm{C}$-arm positions are needed to align the needle to a target structure labeled by the surgeon in both X-ray images. The actual alignment is done by the surgeon while she/he sees the alignment of the needle to the target structure on video images co-registered with the X-ray image. Preliminary experimental results show the power of CAMC for medical augmented reality imaging. It also shows that this imaging system can provide surgeons with new possibilities for image guided surgery. In particular it reduces the X-ray exposures to both patient and physician.
\end{abstract}

\section{Introduction}

This paper describes an intra-operative video-augmented imaging system, introduced in 11, and presents an exemplary use of this system for precise needle placement. The system provides real-time co-registered X-ray fluoroscopy and video images. The physician could observe X-ray and video image from the same vantage point, and/or an augmented reality image merging X-ray and optical images in real-time. Assuming that the patient remains immobilized, one X-ray image may be taken, and from that point on, the procedure may be continued under video guidance This allows the surgeon to orient a surgical tool under video imaging in regard to patient's anatomy. This new imaging system has many applications, for example in orthopedic neck surgery (Fig. 1).

In this paper we first describe the design, calibration and construction of the combined video and X-ray fluoroscopy imaging system. This system is based on 

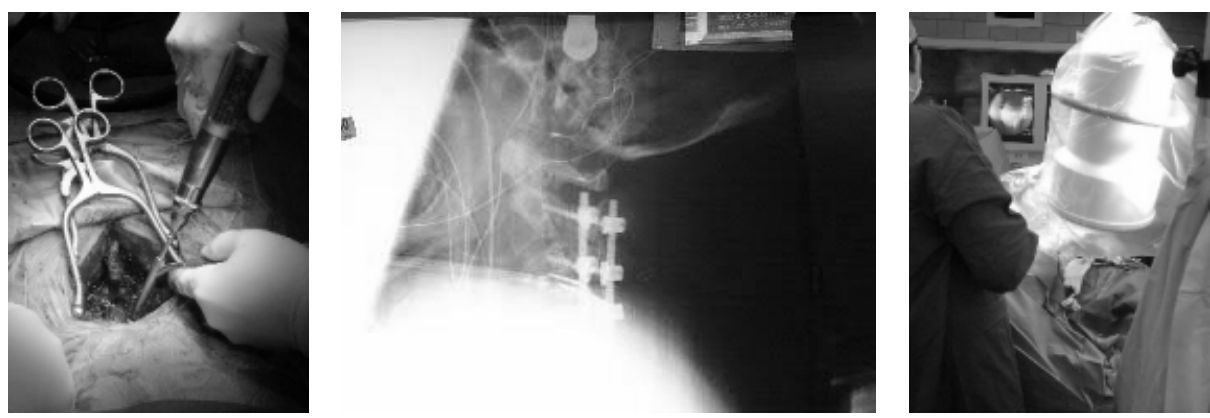

Fig. 1. Orthopedic neck surgery often requires placement of several lateral mass and pedical screws. The potential for this work is the following: Perfect registration between the X-ray and video enables the surgeon to use video to orient the surgical tools for the placement procedures. This allows the physician to use image guidance through out the procedure while taking a reduced number of X-ray images.

the camera augmented mobile C-arm, which was introduced in 12 . A CCD camera attached to the X-ray frame was used to compute the motion of the C-arm to be used for online geometrical calibration. Here, we propose to use the attached CCD camera for augmented reality visualization. Real-time blending of X-ray and video images is not possible unless both system share the same projection geometry. Introducing a double mirror system the optical axes of both imaging systems (X-ray and optical) can be aligned, see [11. With this alignment both images can be merged or co-registered by a simple planar transformation. This allows a real-time augmented reality visualization of mixed X-ray and video images. Figure 2 illustrates the basic design of this system.

This technique of merging live camera images with X-ray fluoroscopic images bears large potential for a number of medical applications. In this paper we take the particular application of needle placement for percutaneous procedures. Minimal invasive surgical techniques are rapidly growing in popularity, among them percutaneous surgical procedures. In comparison with traditional open techniques they reduce patient recovery time and discomfort. However, during these interventions, the surgeon must rely on indirect views on the patient's anatomy provided by imaging modalities such as X-ray fluoroscopy. X-ray systems provide the surgeon with images, which are $2 \mathrm{D}$ projection of $3 \mathrm{D}$ anatomies. This is in contrast to open surgery, where the physician has a direct view (although partial) of the patient's anatomy.

Targeting deep seated anatomic structures using only X-ray projections is challenging and requires experienced physicians. Many researchers propose to register patient's pre-operative CT data with intra-operative X-ray fluoroscopic images to help the physician. Others propose to register the pre-operative CT reconstruction with the patient and an external tracking system for navigation and guidance, e.g. see [8, 1, 6]. Although a wide range of possible imaging modalities, like CT, US, MRI, and even combinations of them, is available for percutaneous procedures, X-ray fluoroscopy remains the most frequently used modality. This 


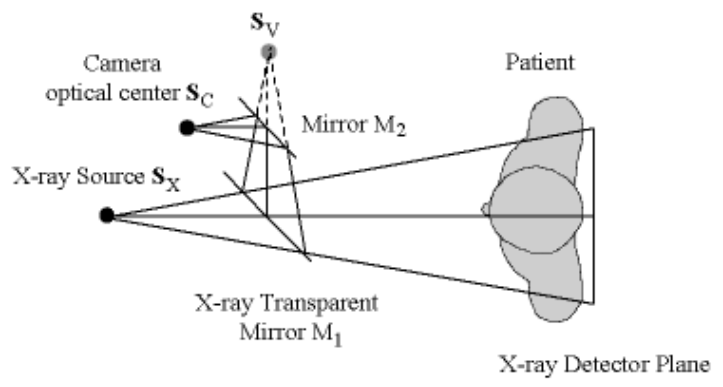

Fig. 2. Conceptual Design: A double mirror system is used to align the optical axes of X-ray and optical imaging system

may be caused by the simple fact that mobile X-ray systems and C-arm fluoroscopes are low cost, readily available in most hospitals, and they lend themselves to practical use in the operating room.

The following section introduces the design and mathematical framework of the real-time X-ray and optical image overlay (CAMC). It also describes the calibration procedure which needs to be performed only during the original construction of the CAMC system. Section 3 presents preliminary experimental results. Our new needle placement procedure is then described in section 4 The work is summarized and future work is discussed in section 5 .

\section{Mathematical Framework for Real-Time X-Ray and Optical Image Overlay}

\subsection{General Concept}

Merging X-ray and optical images requires that both images are taken from the same viewpoint, which is physically impossible. Nevertheless, with the help of two mirrors the camera and the X-ray source can be positioned relative to each other such that both optical axes become aligned [11]. This is visualized in Fig. 3]

Due to different focal lengths of both imaging systems it is not necessary to bring both optical centers to virtually the same point. The different images are then scaled before merging, with the scale factor determined during an offline calibration procedure.

During the $\mathrm{C}$-arm motion the intrinsic geometry of the X-ray imaging system (relation between $\mathrm{X}$-ray source and detector) will not be constant due to the weights of both X-ray source and detector and the thereby caused minor bending of the C-arm. This has an influence on the optical axis of the X-ray system, which is defined as the normal dropping from the X-ray source onto the detector plane. This effect makes it impossible to calibrate the system, because the camera position would have to be different for every $\mathrm{C}$-arm position. To overcome this 

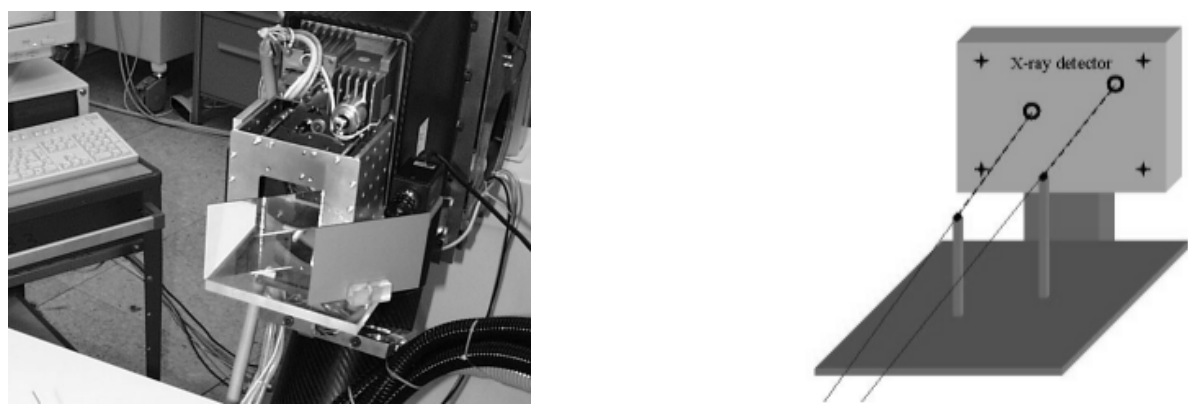

Fig. 3. X-ray source, optical camera attached to it, and the double mirror system used to align the two imaging geometry (left), and the calibration setup (right): on-plane markers (crosses), off-plane markers (spheres) and corresponding on-plane markers (rings) - see text for details.

limitation we use the "virtual detector" concept proposed in [12]. A small number of X-ray opaque markers are arranged on a plate that is attached to the X-ray source such that the projected markers appear on the image close to the border. A planar transformation is applied to the image to bring the projected markers to pre-defines positions. This in effect corrects for the variations in the intrinsic parameters of the X-ray system.

\subsection{Setup and Calibration Procedure}

In order for our system to work properly as described in the previous section we need

1. Precise positioning of the camera and the two mirrors in order to align both optical axes

2. 2D-2D co-registration (in-plane rotation, translation, and scale) that corrects for the differences in intrinsic parameters of both imaging systems.

Both requirements are satisfied with a single calibration task. The two mirrors are fixed as shown in Fig.3. We use a set of marker objects that are visible to both X-ray and optical camera. Four (or more) of them are placed on the X-ray detector. They are used to estimate the planar transformation that co-registers both images in $2 \mathrm{D}^{1}$. Two (or more) markers are placed visible for both imaging systems with a reasonable distance (e.g. about $5 \mathrm{~cm}$ ) to the X-ray detector. Both optical axes are aligned if a single planar transformation computed from the onplane markers also brings the projections of the off-plane markers to a match. The calibration task now is to bring the camera in a position such that this requirement is satisfied. The following algorithm is proposed:

${ }^{1}$ Here, we consider mobile C-arms equipped with a Solid State Detector with no distortion artifact. Distortion correction is necessary for traditional systems [4, 7]. 


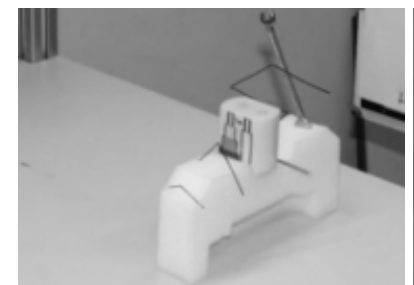

(a) setup

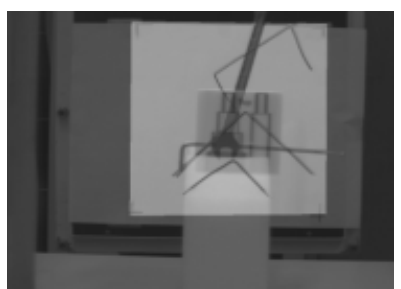

(d) $50 \%-50 \%$

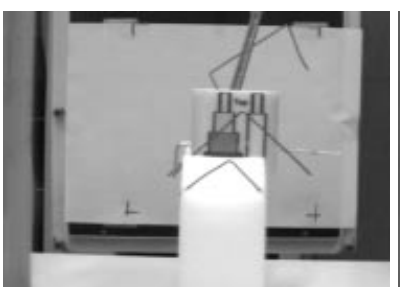

(b) $100 \% \mathrm{CCD}$

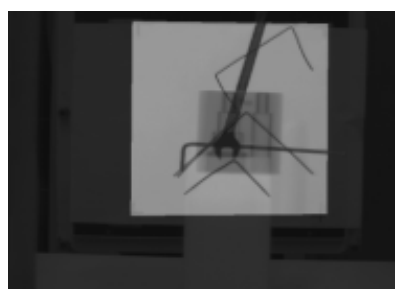

(e) $25 \%-75 \%$

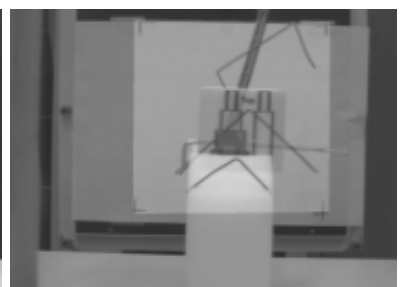

(c) $75 \%-25 \%$

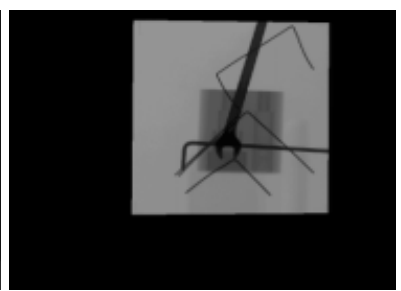

(f) $100 \%$ X-ray

Fig. 4. Experimental results: setup (a), original video (b) and X-ray (f), and result of overlay for 3 different opacities (c-e).

1. Attach four or more markers on the X-ray detector (on-plane markers), and position two or more marker objects (off-plane markers) in the view of both imaging systems.

2. For each off-plane marker attach an extra marker on the detector plane such that all these pairs of markers appear superimposed in the X-ray image (see Fig.3).

3. Modify the camera's position until the pairs of off-plane and corresponding on-plane markers are also superimposed on the video image.

4. Fix the camera and estimate the planar transformation using all on-plane markers.

The system will then be fully calibrated, and can provide real-time overlay of $\mathrm{X}$-ray and optical images. Note that this calibration procedure is done only once by the C-arm manufacturer. No extra calibration is needed before or during the operation.

\section{Preliminary Experimental Results}

Here we first want to present some results that, in general, demonstrate the power of camera augmented mobile $\mathrm{C}$-arm with the double mirror system. Then we describe a simple experiments focused at a needle placement procedure in a lab setup. So far we did not conduct real experiments that would result in accuracy measures. This is due to the fact that the placement procedure depends on the person performing it. We will have to further discuss this application with surgeons in order to conduct a large number of experiments and find out how best to evaluate the accuracy of such alignment procedure. 

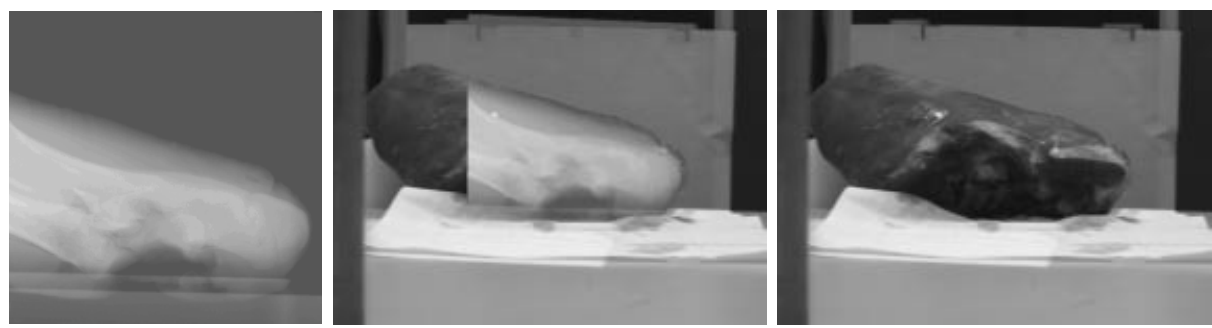

Fig. 5. Experimental results: X-ray image (left), video image (right), and overlaid optical and X-ray image of pig's knuckle (center).

\subsection{Augmented Reality Visualization with CAMC}

In this experiment we use a set of different metallic objects placed on the table as visualized in Fig. 4 The objects are placed on the table such that they occlude each other. The occluded parts can then be "seen" in the combined optical and X-ray image, see Fig.4. The "visibility" can be adjusted smoothly from pure $\mathrm{X}$-ray to pure optical vision.

The result of another experiment that is closer to an actual medical application is visualized in Fig.5. A pig's knuckle is positioned on the table. The X-ray image (left) shows the bone structure. In the combined optical and X-ray image (right) the bone structure can be seen on top of the optical image of the leg.

\section{Needle Placement under AR Visualization}

\subsection{Needle Placement Procedures - State of the Art}

Different needle placement techniques have been proposed in the literature. The axial aiming technique [5, 13, is a well established method for needle placement under X-ray fluoroscopy. The fluoroscope is positioned such that needle tip and target point become superimposed on the X-ray image during the whole insertion of the needle into the body. However, this positioning is not easy and requires much X-ray exposure. In addition, the depth of insertion cannot be observed from the axial view.

Different mechanical manipulators such as PAKY and LARS robot were introduced and used for needle injection under radiological guidance by 15, 16, and 14, 3, 2, The design of these robots is excellent and they have proven to be precise and well adopted for their use in operating room. However, the needle placement methods they propose all require time consuming pre-operative registration procedures between the robot, imaging system and the patient's anatomy. Recently visual servoing techniques have been proposed and tested on some of these robots for automatic needle placement [9] 10]. By taking advantage of projective geometry and projective invariants the needle is aligned automatically from a small number of X-ray images taken from two different C-arm positions. 


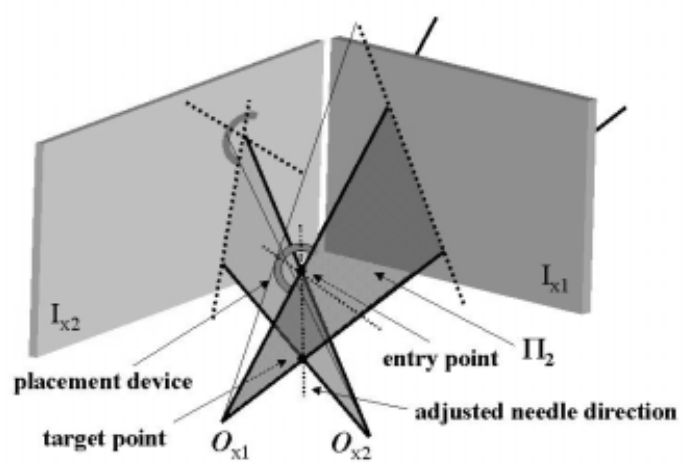

Fig. 6. 3D alignment from two perspective views: Each viewing plane includes both entry and target points as well as their projections on the respective image planes. The intersection of both planes defines the $3 \mathrm{D}$ orientation of the needle.

Here we show that when using our combined X-ray and video imaging system, referred to as CAMC, the necessary radiation may be decreased to a minimum of two X-ray images. Additional X-ray images might be taken to verify the accuracy of the alignments. This augmented reality imaging system can be used in conjunction with all existing methods. They can all take advantage of this system to reduce the X-ray exposure. Here we describe a method, where no motorized robotic device is used. This can be considered both as a practical method and as a proof of concept.

\subsection{Placement Technique}

The direction of the needle will be determined from only two X-ray images from different positions of the C-arm. For each of the two views we are able to determine a plane that includes both the entry point of the needle and the target point. The orientation of the needle in $3 \mathrm{D}$ is then defined by the intersection of these two planes.

A plane that includes both entry and target point is the one defined by the projection of both points in the image plane and the optical center, see Fig.6. For the first view such a plane is determined. With the C-arm in the second viewing position the motion of the needle is restricted to the plane determined before. This ensures that when the needle is aligned for the second view, the final 3D alignment is accomplished.

This method requires rotations of the needle in restricted planes. A placement device such as the prototyp pictured in Fig. 7 becomes necessary. Planes $\Pi_{1}$ and $\Pi_{3}$ are perpendicular to each other. Plane $\Pi_{2}$ can be rotated around the normal of $\Pi_{3}$ (axis $a_{3}$ ). Both planes together can be rotated around the normal of plane $\Pi_{1}$ (axis $a_{1}$ ). The needle can be rotated around the normal of plane $\Pi_{2}$ (axis $\left.a_{2}\right)$. 


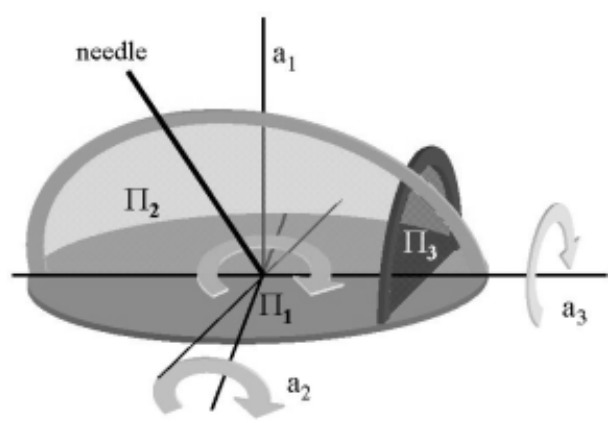

(a) general concept

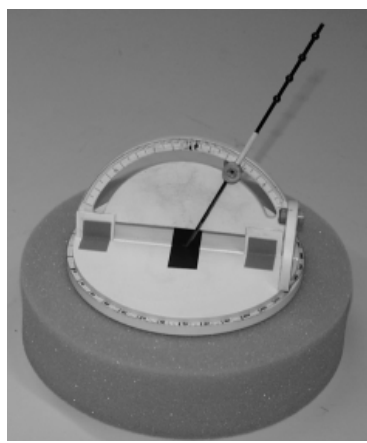

(b) used prototype

Fig. 7. Placement device: General concept (a) and lab setup prototype (b).

\subsection{Placement Procedure}

With the $\mathrm{C}$-arm in the first position the goal is to orient the placement device and $\mathrm{C}$-arm such that the needle, the optical(or X-ray) center, the entry point, and the target point become coplanar. This is accomplished by aligning axis $a_{3}$, which is included in plane $\Pi_{2}$, with the X-ray source. This can be done by moving the C-arm until $a_{3}$ is visualized as a single point on the optical image. This makes the optical center and the entry point, which lies on $a_{3}$, coplanar with $\Pi_{2}$. The projected plane $\Pi_{2}$ appears now as a line $l_{2}$, whereas $a_{3}$ is projected onto a point. The next step is then to rotate $\Pi_{2}$ around $a_{3}$ in order to make the target point aligned with $l_{2}$. The target point now lies on $\Pi_{2}$. This plane includes both entry and target point as well as the optical center. This plane is all that can be determined from the first view. The orientation of plane $\Pi_{2}$ relative to planes $\Pi_{1}$ and $\Pi_{3}$ is fixed and the $\mathrm{C}$-arm is brought into a second position for which the optical center is not coplanar to plane $\Pi_{2}$. The alignment of the needle and the target in plane $\Pi_{2}$ is then accomplished. The needle is rotated around $a_{2}$ until entry point, target point, and the optical center become coplanar. See Fig. 6 for a visualization. Summarizing this leads to the following algorithm:

\section{First C-arm position}

1. Position needle placement device at the entry point.


2. Align axis $a_{3}$ and camera optical center by turning placement device around axis $a_{1}$ and moving $\mathrm{C}$-arm while observing the video image.

3. Take first X-ray image and select target point.

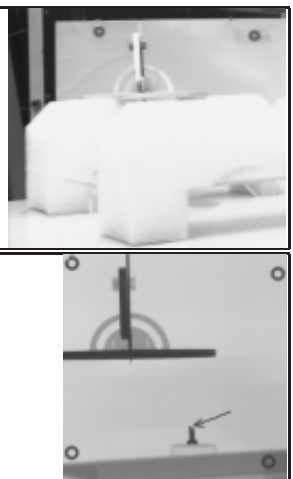

4. Merge X-ray and video image.
$\begin{aligned} & \text { Target point becomes visible in the } \\ & \text { video image. }\end{aligned}$

5. Rotate plane $\Pi_{2}$ about $a_{3}$ under video control until virtual extension of needle becomes aligned with target point. Fix orientation of plane $\Pi_{2}$ relative to planes $\Pi_{1}$ and $\Pi_{3}$.

\begin{tabular}{l}
\hline Second C-arm position \\
6. Bring C-arm into second position. \\
7. Take second X-ray image and select target point. \\
\hline \hline 8. Merge X-ray and CCD image. \\
Target point becomes visible in \\
video image.
\end{tabular}


9. Rotate needle under video control around axis $a_{2}$ until virtual extension of needle becomes aligned with target point.

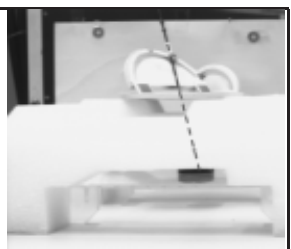

The needle is now completely aligned with the target in 3D. The insertion depth of the needle can be estimated using projective invariants as proposed in [9, 10 .

\section{Conclusion}

This paper presents a simple method for semi-automatic placement of a surgical instrument such as a needle under a new augmented reality visualization system [11. This imaging system includes an X-ray C-arm, equipped with a CCD camera that is attached to the X-ray source, and a double mirror system. The cameras and the double mirror system are installed and calibrated such the optical axes of both imaging systems are aligned. The X-ray and optical images are then coregistered using a simple planar transformation. This new imaging system results in real-time overlay of X-ray and CCD camera images. This allows the surgical operations that need intra-operative fluoroscopic imaging to be done under X-ray augmented video instead. Only a single X-ray is needed for each C-arm position. Deep-seated target structure marked in the X-ray image is by construction coregistered in the CCD camera image. The paper describes a simple interactive method to align a needle with the target under X-ray augmented video guidance. A minimum number of two X-ray images are needed for the alignment procedure. Additional X-ray images might be taken to verify the final 3D alignment from additional views. In order to evaluate the efficiency of needle placement under augmented reality visualization, one could run a series of experiments. In these experiments the physicians would first try to align the needle with the deepseated target using X-ray fluoroscopic guidance. They would then use X-ray augmented video guidance to achieve this alignment.

\section{Acknowledgements}

We would like to thank Dr. Lee H. Riley III, M.D., at Johns Hopkins University Hospital who allowed us to observe several surgical procedures using X-ray and X-ray fluoroscopy. We also thank Bernhard Geiger for useful comments.

\section{References}

[1] Medtronic. http://www.sofamordanek.com/fluoronav/fluoro.htm.

[2] A. Bzostek, A. C. Barnes, R. Kumar, J. H. Anderson, and R. H. Taylor. A testbed system for robotically assisted percutaneous pattern therapy. In First International Conference on Medical Image Computing and Computer-Assisted Intervention (MICCAI), Cambridge, UK, 1999. 
[3] A. Bzostek, S. Schreiner, A. C. Barnes, J. A. Cadeddu, W. W. Roberts, J. H. Anderson, R. H. Taylor, and L. Kavoussi. An automated system for precise percutaneous access of the renal collecting system. In Proceedings of the First joint Conference on CVRMed-MRCAS, Grenoble, France, 1997.

[4] R. Fahrig, M. Moreau, and D. W. Holdsworth. Three-dimensional computer tomographic reconstruction using a C-arm mounted XRII: Correction of image intensifier distortion. Medical Physics, 24:1097-1106, 1997.

[5] R. Greene. Transthoracic needle aspiration biopsy. In Interventional Radiology, Ed. C. A. Athanasoulis, R. C. Pfister and J. Saunders, pages 587-634, 1982.

[6] W. E. L. Grimson, M. E. Leventon, G. Ettinger, A. Chabrerie, F. Ozlen, S. Nakajima, H. Atsumi, R. Kikinis, and P. Black. Clinical experience with a high precision image-guided neurosurgery system. In First International Conference on Medical Image Computing and Computer-Assisted Intervention (MICCAI), pages 63-73, 1998.

[7] E. Gronenschild. The accuracy and reproducibility of a global method to correct for geometric image distortion in the X-ray imaging chain. Medical Physics, 24:1875-1888, 1997.

[8] S. Lavallee, P. Sautot, J. Troccaz, P. Cinquin, and P. Merloz. Computer assisted spine surgery: a technique for accurate transpedicular screw fixation using CT data and a 3D optical localizer. In First International Symposium on Medical Robotics and Computer-Assisted Surgery (MRCAS), pages 315-322, Pittsburgh, PA, USA, 1994.

[9] M. H. Loser, N. Navab, B. Bascle, and R. H. Taylor. Visual servoing for automatic and uncalibrated percutaneous procedures. In Proceedings of SPIE Medical Conference, San Diego, California, USA, February 2000.

[10] N. Navab, B. Bascle, M. H. Loser, B. Geiger, and R. H. Taylor. Visual servoing for automatic and uncalibrated needle placement for percutaneous procedures. In Proceedings of the IEEE Conference on Computer Vision and Pattern Recognition, Hilton Head Island, SC, USA, June 2000.

[11] N. Navab, M. Mitschke, and A. Bani-Hashemi. Merging visible and invisible: Two camera-augmented mobile C-arm (CAMC) applications. In IEEE International Workshop on Augmented Reality, pages 134-141, San Francisco, CA, USA, 1999.

[12] N. Navab, M. Mitschke, and O. Schütz. Camera augmented mobile C-arm (CAMC) application: 3D reconstruction using a low-cost mobile C-arm. In Second International Conference on Medical Image Computing and Computer-Assisted Intervention (MICCAI), pages 688-697, Cambridge, England, 1999.

[13] J. H. Newhouse and R. C. Pfister. Renal cyst puncture. In Interventional Radiology, Ed. C. A. Athanasoulis, R. C. Pfister and J. Saunders, pages 409-425, 1982.

[14] S. Schreiner, J. H. Anderson, R. H. Taylor, J. Funda, A. Bzostek, and A. C. Barnes. A system for percutaneous delivery of treatment with a fluoroscopically guided robot. In Proceedings of the First joint Conference on CVRMed-MRCAS, pages 747-756, Grenoble, France, 1997.

[15] D. Stoianovici, J. A. Cadeddu, R. D. Demares, H. A. Basile, R. H. Taylor, L. L. Whitcomb, W. N. Sharpe Jr., and L. Kavoussi. An efficient needle injection technique and radiological guidance method for percutaneous procedures. In Proceedings of the First joint Conference on CVRMed-MRCAS, Grenoble, France, 1997.

[16] D. Stoianovici, L. L. Whitcomb, J. H. Anderson, R. H. Taylor, and L. R. Kavoussi. A modular surgical robotic system for image guided percutaneous procedures. In First International Conference on Medical Image Computing and ComputerAssisted Intervention (MICCAI), Cambridge, MA, USA, 1998. 